\title{
Estimation of ground state pentaquark masses
}

\author{
K. Xu* and N. Ritjoho ${ }^{\dagger}$ \\ School of Physics, Suranaree University of Technology, 111 University Avenue \\ Nakhon Ratchasima 30000, Thailand \\ *gxukai1123@gmail.com \\ †salapao-punjin@hotmail.com \\ S. Srisuphaphon \\ Department of Physics, Burapha University, 169 Long-Hard Bangsaen Road \\ Chonburi 20131, Thailand \\ edsorakrai@gmail.com \\ Y. Yan \\ School of Physics, Suranaree University of Technology, 111 University Avenue \\ Nakhon, Ratchasima 30000, Thailand \\ yupeng@sut.ac.th \\ Published 16 April 2014
}

\begin{abstract}
Permutation groups are applied to analyze the symmetries of multiquark systems and wave functions of pentaquark states are constructed systematically in the language of Yamanouchi basis. We estimate the mass of baryons in the constituent quark model with one-gluon-exchange interaction, assuming that baryons consist of the $q^{3}$ component as well as the $q^{4} \bar{q}$ pentaquark component.
\end{abstract}

Keywords: Pentaquark state; constituent quark model; one-gluon-exchange.

PACS Numbers: 12.39.Jh, 14.20.Pt

\section{Introduction}

Baryon mass spectrum has been studied over decades, but theoretical results are still largely inconsistent with experimental data. No need to mention the higher excited states, even the low-lying resonances, for example, the Roper resonance $N(1440)$, $N(1520)$ and $N(1535)$ have been of problem. Theoretical works in the three-quark picture always predict a larger mass for the Roper resonance $N(1440)$ than for $N(1520)$ and $N(1535)$. It is revealed, in our previous work on the annihilation

This is an Open Access article published by World Scientific Publishing Company. It is distributed under the terms of the Creative Commons Attribution 3.0 (CC-BY) License. Further distribution of this work is permitted, provided the original work is properly cited. 
reactions $\bar{p} p \rightarrow \phi X^{1}$ and also a number of works of others, that the proton may possess a considerable $q^{4} \bar{q}$ component. ${ }^{2,3}$ It is natural to extend this understanding, assuming that baryons consist of the $q^{3}$ component as well as the $q^{4} \bar{q}$ pentaquark component. In this work we consider all possible $q^{4} \bar{q}$ configurations, work out the wave functions with the aid of group theory ${ }^{4,5}$ and then estimate the mass for each configuration of ground state pentaquarks.

\section{Pentaquark Wave Functions}

The construction of the wave function of pentaquarks plays a crucial role in the present work. It is not straightforward to construct the wave function of pentaquark states since the permutation symmetries of the five-particle system are rather complicated. We apply the Yamanouchi basis approach systematically to construct $\psi\left(q^{4}\right)$ systems first.

The algebraic structure of pentaquark states consists of the usual spin-flavor and color algebras $S U_{s f}(6) \otimes S U_{c}(3)$ with $S U_{s f}(6)=S U_{f}(3) \otimes S U_{s}(2)$. The permutation symmetry of the four-quark configuration of pentaquark states is characterized by the $S_{4}$ Young tabloids [4], [31], [22], [211] and [1111]. That the pentaquark should be a color singlet demands that the color part of the pentaquark wave function must be a $[222]_{1}$ singlet. Since the color part of the antiquark in pentaquark states is a $[11]_{3}$ antitriplet, the color wave function of the four-quark configuration must be a $[211]_{3}$ triplet. That the total wave function of the four quark configuration is antisymmetric implies that its orbital-spin-flavor part must be a [31] state. The total wave function of the $q^{4}$ configuration may be written in the general form

$$
\psi=\sum_{i, j=\lambda, \rho, \eta} a_{i j} \psi_{[211]_{i}}^{c} \psi_{[31]_{j}}^{o s f},
$$

The coefficients can be determined by applying the permutations (12), (23) and (34) of the $S_{4}$ group to Eq. (1), and a fully antisymmetric wave function is derived for the $q^{4}$ configuration,

$$
\psi=\frac{1}{\sqrt{3}}\left(\psi_{[211]_{\lambda}}^{c} \psi_{[31]_{\rho}}^{o s f}-\psi_{[211]_{\rho}}^{c} \psi_{[31]_{\lambda}}^{o s f}+\psi_{[211]_{\eta}}^{c} \psi_{[31]_{\eta}}^{o s f}\right),
$$

The color wave function of the $q^{4}$ configuration, in Eq. (2), can be derived by applying the [211] $\lambda$-type, $\rho$-type and $\eta$-type projection operators of the $S_{4}$ group to an arbitrary four-quark color state, and the singlet color wave function $\Psi_{[211]_{j}}^{c}$ $(j=\lambda, \rho, \eta)$ in Eq. (2) is given by

$$
\Psi_{[211]_{j}}^{c}=\frac{1}{\sqrt{3}}\left[\psi_{[211]_{j}}^{c}(R) \bar{R}+\psi_{[211]_{j}}^{c}(G) \bar{G}+\psi_{[211]_{j}}^{c}(B) \bar{B}\right],
$$

where $\psi_{[211]_{j}}^{c}(G)$ and $\psi_{[211]_{j}}^{c}(B)$ are the color $G$ and $B$ wave functions of the $q^{4}$ cluster, derived by acting the projection operators on the sequences $R R G B, G G R B$ 
and $B B G R$, respectively. The projection operators are derived from the [211] representation of the $S_{4}$ group in the Yamanouchi basis,

$$
\begin{aligned}
P_{[211]_{\lambda}} & =\sum_{i=1}^{24}\left\langle[211](3211)\left|P_{i}\right|[211](3211)\right\rangle P_{i}, \\
P_{[211]_{\rho}} & =\sum_{i=1}^{24}\left\langle[211](3121)\left|P_{i}\right|[211](3121)\right\rangle P_{i}, \\
P_{[211]_{\eta}} & =\sum_{i=1}^{24}\left\langle[211](1321)\left|P_{i}\right|[211](1321)\right\rangle P_{i},
\end{aligned}
$$

where $|[211](3211)\rangle,|[211](3121)\rangle$ and $|[211](1321)\rangle$ are respectively the $\lambda$-type, $\rho$ type and $\eta$-type Yamanouchi basis functions. The summary is over all the permunations of the $S_{4}$ group. By applying the operators on $R R G B$, we get

$$
\begin{aligned}
\psi_{[211]_{\lambda}}^{c}(R)= & \frac{1}{\sqrt{16}}(2|R R G B\rangle-2|R R B G\rangle-|G R R B\rangle-|R G R B\rangle-|B R G R\rangle \\
& -|R B G R\rangle+|B R R G\rangle+|G R B R\rangle+|R B R G\rangle+|R G B R\rangle), \\
\psi_{[211]_{\rho}}^{c}(R)= & \frac{1}{\sqrt{48}}(3|R G R B\rangle-3|G R R B\rangle+3|B R R G\rangle-3|R B R G\rangle+2|G B R R\rangle \\
& -2|B G R R\rangle-|B R G R\rangle+|R B G R\rangle+|G R B R\rangle-|R G B R\rangle), \\
\psi_{[211]_{\eta}}^{c}(R)= & \frac{1}{\sqrt{6}}(|B R G R\rangle+|R G B R\rangle+|G B R R\rangle \\
& -|R B G R\rangle-|G R B R\rangle-|B G R R\rangle) .
\end{aligned}
$$

For ground-state pentaquarks, all five quarks are in the S-wave and form a fully symmetric spatial wave function, and hence the total wave function is easily derived as

$$
\Psi=\frac{1}{\sqrt{3}}\left[\Psi_{[5]}^{o}\left(\Psi_{[211]_{\lambda}}^{c} \Psi_{[31]_{\rho}}^{s f}-\Psi_{[211]_{\rho}}^{c} \Psi_{[31]_{\lambda}}^{s f}+\Psi_{[211]_{\eta}}^{c} \Psi_{[31]_{\eta}}^{s f}\right)\right]
$$

where the subscripts [211] and [31] are for the $q^{4}$ configuration. There are a large number of spin and flavor representations resulting in the [31] spin-flavor configuration.

The spin-flavor wave functions for the [31] configuration can be written in the general forms (the spin part can not be in the [211] and [1111] types while the flavor part can not be in the [1111] type),

$$
\Psi_{[31]}^{s f}=\left(\psi_{[4]}^{s}, \psi_{[31]}^{s}, \psi_{[22]}^{s}\right) \otimes\left(\psi_{[4]}^{f}, \psi_{[31]}^{f}, \psi_{[211]}^{f}, \psi_{[22]}^{f}\right) .
$$

It can be proven that seven of the 12 possible combinations give the [31] spin-flavor permutation symmetry, as shown in Table 1. For the flavor [22] and spin [31] case, 
K. Xu et al.

Table 1. Combinations of spin and flavor configurations to [31] spin-flavor symmetries.

\begin{tabular}{lll}
\hline \multicolumn{3}{c}{$[31]_{F S}$} \\
\hline$[31]_{F} \otimes[22]_{S}$ & {$[31]_{F} \otimes[31]_{S}$} & {$[31]_{F} \otimes[4]_{S}$} \\
{$[211]_{F} \otimes[22]_{S}$} & {$[211]_{F} \otimes[31]_{S}$} & {$[22]_{F} \otimes[31]_{S}$} \\
{$[4]_{F} \otimes[31]_{S}$} & & \\
\hline
\end{tabular}

for example, the spin-flavor wave functions $\Psi_{[31]}^{s f}$ can be derived by assuming

$$
\psi^{s f}=\sum_{i=\lambda, \rho} \sum_{j=\lambda, \rho, \eta} a_{i j} \phi_{[22]_{i}} \chi_{[31]_{j}},
$$

and then applying the $\lambda$-type, $\rho$-type and $\eta$-type projection operators for the representation [31] of the $S^{4}$ group,

$$
\begin{aligned}
\psi_{[31]_{\rho}}^{s f} & =-\frac{1}{2} \phi_{[22]_{\rho}} \chi_{[31]_{\lambda}}-\frac{1}{2} \phi_{[22]_{\lambda}} \chi_{[31]_{\rho}}+\frac{1}{\sqrt{2}} \phi_{[22]_{\rho}} \chi_{[31]_{\eta}}, \\
\psi_{[31]_{\lambda}}^{s f} & =-\frac{1}{2} \phi_{[22]_{\rho}} \chi_{[31]_{\rho}}+\frac{1}{2} \phi_{[22]_{\lambda}} \chi_{[31]_{\lambda}}+\frac{1}{\sqrt{2}} \phi_{[22]_{\lambda}} \chi_{[31]_{\eta}}, \\
\psi_{[31]_{\eta}}^{s f} & =\frac{1}{\sqrt{2}} \phi_{[22]_{\rho}} \chi_{[31]_{\rho}}+\frac{1}{\sqrt{2}} \phi_{[22]_{\lambda}} \chi_{[31]_{\lambda}} .
\end{aligned}
$$

The flavor and spin wave functions, $\phi_{[22]}$ and $\chi_{[31]}$ can be derived the same way as for the color wave function but using the [22] and [31] projectors, respectively. For the spin wave functions one applies the $\lambda$-type, $\rho$-type and $\eta$-type projection operators for the representation [31] of the $S_{4}$ group and derive,

$$
\begin{aligned}
& P_{[31]_{\eta}}(\uparrow \uparrow \uparrow \downarrow) \Longrightarrow \chi_{[31]_{\eta}}(1,1)=\frac{1}{2 \sqrt{3}}|3 \uparrow \uparrow \uparrow \downarrow-\downarrow \uparrow \uparrow \uparrow-\uparrow \downarrow \uparrow \uparrow-\uparrow \uparrow \downarrow \uparrow\rangle, \\
& P_{[31]_{\rho}}(\uparrow \downarrow \uparrow \uparrow) \Longrightarrow \chi_{[31]_{\rho}}(1,1)=\frac{1}{\sqrt{2}}|\uparrow \downarrow \uparrow \uparrow-\downarrow \uparrow \uparrow \uparrow\rangle, \\
& P_{[31]_{\lambda}}(\uparrow \uparrow \downarrow \uparrow) \Longrightarrow \chi_{[31]_{\lambda}}(1,1)=\frac{1}{\sqrt{6}}|2 \uparrow \uparrow \downarrow \uparrow-\downarrow \uparrow \uparrow \uparrow-\uparrow \downarrow \uparrow \uparrow\rangle .
\end{aligned}
$$

The total spin wave function of the pentaquark states is formed by combining the spin wave functions above with the one of the antiquark, that is

$$
\begin{aligned}
\chi\left(q^{4} \bar{q}\right)_{[31]_{\alpha}}= & \sqrt{\frac{2}{3}} \chi_{[31]_{\alpha}}\left(s_{q^{4}}=1, m_{q^{4}}=1\right) \chi_{\bar{q}}(-1 / 2) \\
& -\sqrt{\frac{1}{3}} \chi_{[31]_{\alpha}}\left(s_{q^{4}}=1, m_{q^{4}}=0\right) \chi_{\bar{q}}(1 / 2),
\end{aligned}
$$

with $\alpha=\rho, \lambda, \eta$. The states with other values of the projection $m_{s}$ can be obtained the same way. 
The flavor wave functions are derived by acting $P_{\lambda, \rho}$ on any $q^{4}$ state, for example,

$$
\begin{aligned}
& P_{\rho}(u u d d) \Longrightarrow \phi_{[22]_{\rho}}=\frac{1}{2}(d u d u-d u u d+u d u d-u d d u) \\
& P_{\lambda}(u u d d) \Longrightarrow \phi_{[22]_{\lambda}}=\frac{1}{2 \sqrt{3}}(d u u d+u d u d-2 u u d d+u d d u+d u d u-2 d d u u) .
\end{aligned}
$$

Pentaquark flavor wave functions are given by

$$
\Phi_{[22]_{\rho}}=\phi_{[22]_{\rho}} \bar{q}, \quad \Phi_{[22]_{\lambda}}=\phi_{[22]_{\lambda}} \bar{q} .
$$

\section{Estimation of Masses of Ground State Pentaquarks}

We propose to study the baryon spectrum, assuming that baryons consist of the $q^{3}$ component as well as the $q^{4} \bar{q}$ pentaquark component, where the wave function of baryons may take the form,

$$
|N\rangle=A\left|q^{3}\right\rangle+B\left|q^{4} \bar{q}\right\rangle
$$

where $A^{2}+B^{2}=1$. The baryon mass may be estimated,

$$
M=A^{2} M\left(q^{3}\right)+B^{2} M\left(q^{4} \bar{q}\right),
$$

with $M\left(q^{3}\right)$ and $M\left(q^{4} \bar{q}\right)$ being the mass of the $q^{3}$ and $q^{4} \bar{q}$ systems, respectively,

$$
\begin{array}{r}
M\left(q^{3}\right)=3 m_{q}+N_{q^{3}} \varepsilon_{3}+\Delta m\left(q^{3}\right), \\
M\left(q^{5}\right)=5 m_{q}+N_{q^{4}} \varepsilon^{\varepsilon_{5}}+\Delta m\left(q^{4} \bar{q}\right),
\end{array}
$$

where $m_{q}$ is the constituent mass of the quarks, $N=2 n+l$ with $n$ and $l$ standing for the principle quantum number and orbital angular momentum, $\varepsilon_{3}$ and $\varepsilon_{5}$ are for the harmonic oscillator unit energies. The color-magnetic interaction (or gluon exchange) contributions ${ }^{6}$ take the form,

$$
\begin{gathered}
\Delta m(3 q)=\left\langle\psi\left(q^{3}\right)\left|H_{g}\left(q^{3}\right)\right| \psi\left(q^{3}\right)\right\rangle, \\
\Delta m(5 q)=\left\langle\psi\left(q^{4} \bar{q}\right)\left|H_{g}\left(q^{4} \bar{q}\right)\right| \psi\left(q^{4} \bar{q}\right)\right\rangle,
\end{gathered}
$$

where the color-magnetic interaction is approximated as

$$
H_{g}=-C \sum_{i<j} \frac{\lambda_{i}^{C} \cdot \lambda_{j}^{C}}{m_{i} m_{j}} \vec{\sigma}_{i} \cdot \vec{\sigma}_{j},
$$

where $\lambda_{i}^{C}$ are Gell-Mann matrices in the color space, $\vec{\sigma}_{i}$ are Pauli matrices in the spin space and $m_{i}$ is the constituent mass of the $i$ th quark. 
Taking $q^{3}$ non-strange ground state baryons $N(938)$ and $\Delta(1232)$ as an example where $l=0$ and $n=0$, one gets

$$
\begin{aligned}
& M_{\Delta}=3 m_{q}+8 C_{\chi}, \\
& M_{N}=3 m_{q}-8 C_{\chi},
\end{aligned}
$$

where $N_{q^{3}} \varepsilon_{3}=0, m_{i}=m_{j}=m_{q}$, and $C_{\chi}=\frac{C}{m_{q}{ }^{2}}$ have been applied. Let $M_{\Delta}=1232$ $\mathrm{MeV}$ and $M_{N}=939 \mathrm{MeV}$, we derive the parameters as

$$
m_{q} \approx 361.7(\mathrm{MeV}), \quad C_{\chi}=\frac{C}{m_{q}^{2}} \approx 18.4(\mathrm{MeV}) .
$$

The mass of ground state pentaquarks may be estimated,

$$
M\left(q^{4} \bar{q}\right)=5 m_{q}+\Delta m\left(q^{4} \bar{q}\right)
$$

with

$$
\begin{aligned}
\Delta m\left(q^{4} \bar{q}\right)=\langle & \left.\psi\left(q^{4} \bar{q}\right)\left|H_{g}\left(q^{4} \bar{q}\right)\right| \psi\left(q^{4} \bar{q}\right)\right\rangle, \\
=- & \frac{1}{3} C_{\chi}\left\{\left\langle\psi_{[211]_{\lambda}}^{c}\left|\lambda_{i}^{C} \cdot \lambda_{j}^{C}\right| \psi_{[211]_{\lambda}}^{c}\right\rangle\left\langle\psi_{[31]_{\rho}}^{s f}\left|\vec{\sigma}_{i} \cdot \vec{\sigma}_{j}\right| \psi_{[31]_{\rho}}^{s f}\right\rangle\right. \\
& -2\left\langle\psi_{[211]_{\lambda}}^{c}\left|\lambda_{i}^{C} \cdot \lambda_{j}^{C}\right| \psi_{[211]_{\rho}}^{c}\right\rangle\left\langle\psi_{[31]_{\lambda}}^{s f}\left|\vec{\sigma}_{i} \cdot \vec{\sigma}_{j}\right| \psi_{[31]_{\rho}}^{s f}\right\rangle \\
& +2\left\langle\psi_{[211]_{\lambda}}^{c}\left|\lambda_{i}^{C} \cdot \lambda_{j}^{C}\right| \psi_{[211]_{\eta}}^{c}\right\rangle\left\langle\psi_{[31]_{\rho}}^{s f}\left|\vec{\sigma}_{i} \cdot \vec{\sigma}_{j}\right| \psi_{[31]_{\eta}}^{s f}\right\rangle \\
& +\left\langle\psi_{[211]_{\rho}}^{c}\left|\lambda_{i}^{C} \cdot \lambda_{j}^{C}\right| \psi_{[211]_{\rho}}^{c}\right\rangle\left\langle\psi_{[31]_{\lambda}}^{s f}\left|\vec{\sigma}_{i} \cdot \vec{\sigma}_{j}\right| \psi_{[31]_{\lambda}}^{s f}\right\rangle \\
& -2\left\langle\psi_{[211]_{\rho}}^{c}\left|\lambda_{i}^{C} \cdot \lambda_{j}^{C}\right| \psi_{[211]_{\eta}}^{c}\right\rangle\left\langle\psi_{[31]_{\lambda}}^{s f}\left|\vec{\sigma}_{i} \cdot \vec{\sigma}_{j}\right| \psi_{[31]_{\eta}}^{s f}\right\rangle \\
& \left.+\left\langle\psi_{[211]_{\eta}}^{c}\left|\lambda_{i}^{C} \cdot \lambda_{j}^{C}\right| \psi_{[211]_{\eta}}^{c}\right\rangle\left\langle\psi_{[31]_{\eta}}^{s f}\left|\vec{\sigma}_{i} \cdot \vec{\sigma}_{j}\right| \psi_{[31]_{\eta}}^{s f}\right\rangle\right\} .
\end{aligned}
$$

There are 12 ground state pentaquarks out of the seven possible spin-flavor configurations, as listed in Table 2. After tedious calculations we get an estimation, as shown in Table 2, for the mass of all the 12 ground state pentaquarks. The 12

Table 2. Mass of ground state pentaquarks.

\begin{tabular}{lccc}
\hline Wave function & Spin (or J) & $\Delta m\left(q^{4} \bar{q}\right)$ & $M\left(q^{4} \bar{q}\right)(\mathrm{MeV})$ \\
\hline$\Psi_{[31]_{F S}[211]_{F}[22]_{S}}^{s f}$ & $\frac{1}{2}$ & -294.4 & 1514.1 \\
$\Psi_{[31]_{F S}[211]_{F}[31]_{S}}^{s f}$ & $\frac{1}{2}, \frac{3}{2}$ & $-490.4,-122.4$ & $1318.1,1686.1$ \\
$\Psi_{[31]_{F S}[22]_{F}[31]_{S}}^{s f}$ & $\frac{1}{2}, \frac{3}{2}$ & $-343.2,24.8$ & $1465.3,1833.3$ \\
$\Psi_{[31]_{F S}[31]_{F}[22]_{S}}^{s f}$ & $\frac{1}{2}$ & -48.8 & 1759.7 \\
$\Psi_{[31]_{F S}[31]_{F}[31]_{S}}^{s f}$ & $\frac{1}{2}, \frac{3}{2}$ & $65.3,24.8$ & $1873.8,1833.3$ \\
$\Psi_{[31]_{F S}[31]_{F}[4]_{S}}^{f f}$ & $\frac{3}{2}, \frac{5}{2}$ & $48.8,245.6$ & $1857.3,2054.1$ \\
$\Psi_{[31]_{F S}[4]_{F}[31]_{S}}^{s f}$ & $\frac{1}{2}, \frac{3}{2}$ & $540.0,245.6$ & $2348.5,2054.1$ \\
\hline
\end{tabular}


ground state pentaquarks share the same color configuration term, but the coupling between the spin and flavor configurations are all different and hence the color-magnetic interaction (or gluon exchange) contributions are all different.

\section{Conclusion}

In the work we have applied permutation groups to analyze the symmetries of multiquark systems and constructed wave functions of pentaquark states systematically in the language of Yamanouchi basis. The masses of ground state pentaquarks are estimated in the constituent quark model with one-gluon-exchange interaction.

It is noted that the estimated mass of the pentaquark in the spin-flavor configuration $[31]_{F S}[211]_{F}[22]_{S}$ is very close to the mass of the $N_{1 / 2-}(1535)$.

\section{Acknowledgments}

The work of K.X. was supported by the SUT-PhD/13/2554 Scholarship of Suranaree University of Technology, Nakhon Ratchasima. The work of N.R was supported by the Development and Promotion of Science and Technology Talents Project(DPST).

\section{References}

1. S. Srisuphaphon, Y. Yan, Th. Gutsche, and V.E. Lyubovitskij, Phys. Rev. D 84, 074035 (2011).

2. C.S. An, D.O. Riska, and B.S. Zou, Phys. Rev. C 73,035207 (2006)

3. B.Julia-Diaz and D.O.Riska, Nucl. Phys. A 780. 175-186 (2006).

4. Y. Yan, Applied Group Theory in Physics (Suranaree University of Technology, 2006).

5. Y. Yan and S. Srisuphaphon, Prog. Part. Nucl. Phys. 67, 496 (2012).

6. S. Capstick, and N. Isgur Phys. Rev. D 34, 2809 (1986). 